\title{
Medium Access Guarantee in Wireless Mesh Network
}

\author{
S.Jounaidi \\ Computer, Networks, Mobility \\ and Modeling laboratory \\ FST, Hassan 1st University, \\ Settat, Morocco
}

\author{
Y.Saadi \\ Computer, Networks, Mobility \\ and Modeling laboratory \\ FST, Hassan 1st University, \\ Settat, Morocco
}

\author{
B.Nassereddine \\ Computer, Networks, Mobility \\ and Modeling laboratory \\ FST, Hassan 1st University, \\ Settat, Morocco
}

\begin{abstract}
The wireless mesh network WMN based on the $802.11 \mathrm{~s}$ standard is a new concept that allow to broaden the network coverage in order to maximize the number of client stations, taking advantage of the internet connection.

This technology presents several problems about certain levels of the congestion, especially the non-exploitation of the resource parts caused by the unnecessary rebroadcast and the collision between packets in the queues. As a result, we obtain the unfairness of medium access.

The problem of the rebroadcast of unnecessary packets is controlled by the rebroadcast control mechanisms. One of these mechanisms had been chosen, that in a recent work they studied the capacity of the network that adopt this mechanism .The conclusions of this work, will allow us to define an algorithm to organize the access of flows to the medium, and give more opportunity to the far nodes to occupy a part of the medium.
\end{abstract}

\section{Keywords}

WMN, congestion, CBF, Capacity, Inter-departure.

\section{INTRODUCTION}

The wireless mesh network is a communication infrastructure connecting in a cooperative spirit, a package of a local area wireless networks (WLAN) where the link between access points is also wireless, it is a combination between both infrastructure modes and ad-hoc, where each Mesh Access Point MAP constitute a relay, and we assume that the mesh point MP are not mobile or weakly mobile. The last two components are participating in the transfer of flows from client Stations STA to the Mesh Portal Point MPP and then to internet via a wired infrastructure "fig.l". This type of network is characterized by flexibility, decentralization, covers a large distance and stands fault tolerance (in case of breakdown of a path, the path can easily change). It is also relatively cheaper and responds to temporary or permanent needs of a network connection.

The broadcast by flooding is a method that aims to reach all nodes in the network by a broadcast message to propagate some information "Fig. 2".

The most simple of these methods is the blind flooding where each node blindly rebroadcasts a broadcast message when it is received for the first time. According to "Fig.2", we note that the unnecessary rebroadcasts are participating in the wastage of resources.

This mechanism is strongly used in multi-hop networks. Indeed, it is deployed in a lot of routing protocols such as ZRP, AODV ... and the Hybrid Wireless Mesh Protocol HWMP. It is also used in many applications such as ARP, DHCP ...
The Mac layer of the nodes in wireless mesh networks can receive a large amount of broadcast traffic from neighboring nodes. Those last, deploy applications that use a lot of flooding and also routing protocols that depend on the broadcast.

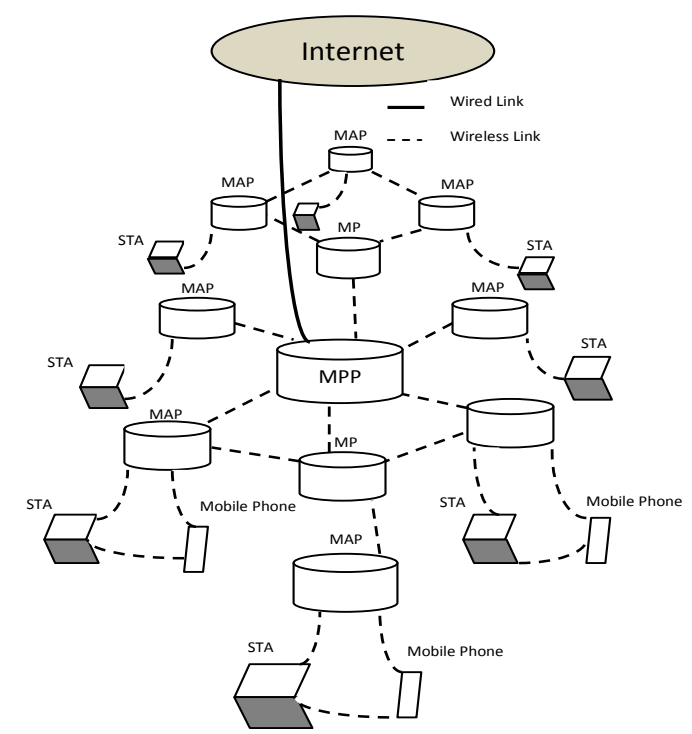

Fig 1: Architecture of a wireless mesh network

This can be fatal for the wireless mesh networks due to the increased probability of collision and contention at the Mac level, thus occupying an amount of bandwidth which negatively affects the capacity of the network.

Several research groups have focused their works on solutions to the problem of unnecessary rebroadcasts. And finally, they proposed several mechanisms of broadcast controls.
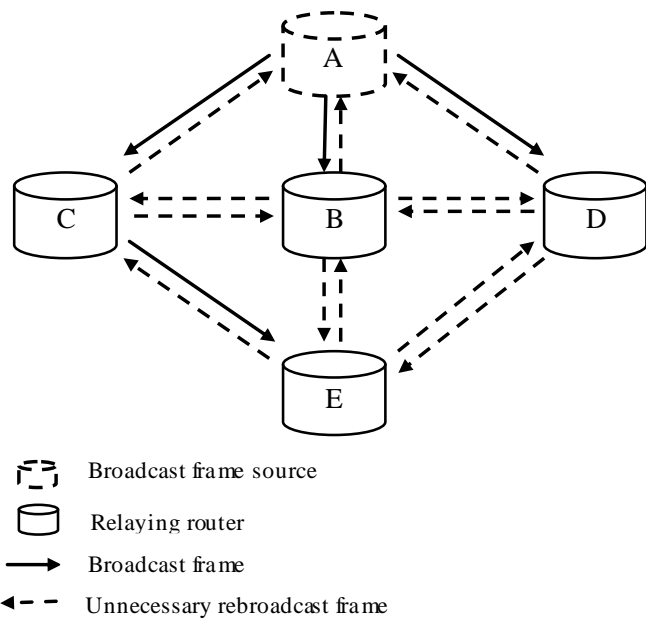

Fig 2: Example of broadcast by flooding 
The wireless mesh network also encounters the problem of collusion about the flows access to the medium from various sources.

For commercial reasons, a working group has focused them research to reform 802.11 to $802.11 \mathrm{~s}$ standard. The goal was to expand the existence of 802.11 standard and his architecture to stand the paradigm of wireless mesh network. The new protocol has to allow to the access points to communicate with each other and form a multi-hop, mesh and wireless architecture. The new architecture produces a concentration of flows to the gateway and consequently an increase of collision between flows to reach gateway.

In this article, we will propose an algorithm that resolves the congestion problem using a mechanism of broadcast control CBF [1].

To define our algorithm, we will use the conclusions that were emerged from the work [2], it was the study of the behavior of the network capacity using the CBF and varying the parameters inter-departure/size of packets.

This research is organized as follows: In the second part, we present the congestion problem. In the section III, we mention some related works about the congestion. Afterward, we will present the conclusions of the CBF study that we chose. Finally, we propose an algorithm that will guarantee the access medium.

\section{RELATED WORKS}

In the context of solving the congestion problem, several researches have been done in order to find mechanisms to guarantee the medium access for different nodes in the wireless mesh network, and therefore guarantee the routing of all packet flows to their destinations. Among this body of work, we specify:

The work done in [3] proposed an algorithm established in each network node, to ensure maximum access to all packets of the different flows in the queue. The executing of the algorithm depends on the information stored in each node, such information concerning the sharing of the queue for flows that traverse a node, and coming from different sources. The information is stored in a table and varies dynamically according to the change of inter-arrival of packet.

The mechanism innovated in the work [4] is effective against the aggressive flows. This mechanism operates by using a table included in each node that stores the information on the aggressive and non-aggressive flows that cross the node. It also uses the value of a variable named "drop_probability" in order that the node decides to reject or accept the packets of aggressive flows. The variable "drop_probability" increases during the rejection of packets coming from the nonaggressive flows by the node to block the packets access from the aggressive flows. Once the non-aggressive flows gain access to the medium, the "drop_probability" decreases.

The working group [5] has considered that the wireless mesh network contains multiple gateways that have the role to transmit data to the internet. In this sense, this working group has shared the gateways into two types: the first type handles the data of high rate, and the second handles the data of low rate. The objective is to separate the aggressive from the nonaggressive flows.

The mechanism proposed in the article [6] had for purpose to resolve to congestion problem using a technique of dynamic routing. This mechanism is based on an algorithm that can at first detect the loss channel due to the congestion at the node queue. Then, if data from a sender node cross this channel, then they must change the path to another alternative since the wireless mesh network stands the fault tolerance.

The authors of [7] have tried to solve the congestion problem by using the $802.11 \mathrm{e}$ protocol, they have limited the reservation time of resource for each node using the TXOP parameter (transmission opportunity). For this purpose, they have allocated a transmission time for each node proportional to the TXOP and depending on the number of client stations associated to this node.

The work [8] was performed to reduce the probability of collision between the flows. The concept is based on a mechanism which allows to allocate to each node a different value of Contention window $\mathrm{CW}$. If we have the same CWmin and CWmax in each node, then we will have a high probability of collision. That's why the mechanism chose to give the smaller values of CWmin and CWmax for the adjacent nodes to the gateway and the higher value to the nodes far from the gateway.

\section{CONGESTION PROBLEM}

The clarification of the congestion problem, necessitate a simulation on NS2 where there is a grid topology $(4 \mathrm{x} 4)$ of a wireless mesh network based on 802.11 s standard "fig. 3 ". The access points communicate between them using the interfaces of 802.11 standard to form a wireless backbone. The transmission range for each node has been set at 250 meters. The distance between each node is 175 meters, which guarantee that the nodes cannot reach the distant nodes without passing through adjacent nodes. Each node connected to its own stations. The channels are configured to have a data rate of $11 \mathrm{Mbps}$ and a base rate of $2 \mathrm{Mbps}$.

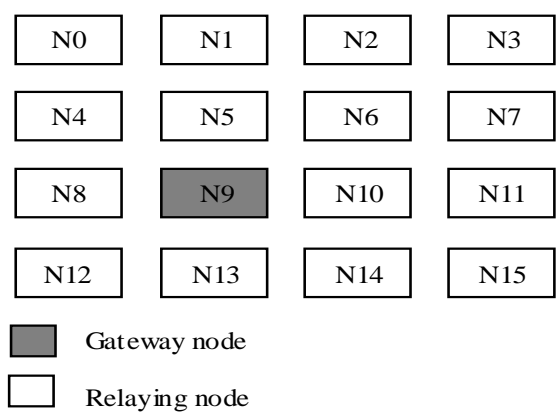

Fig 3: Grid topology (4x4) of WMN

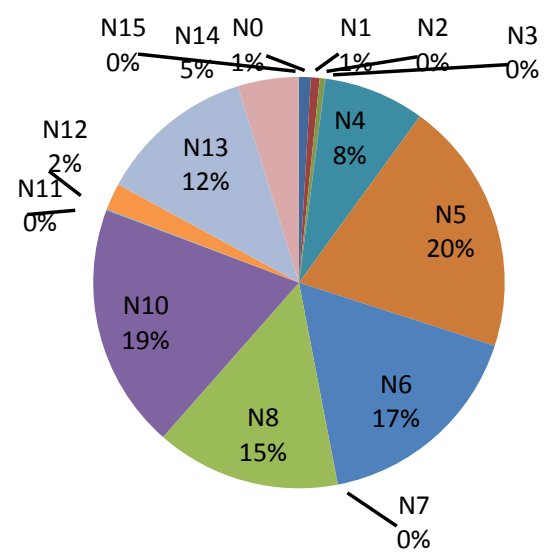

Fig 4: The percentage of the number of packets received by gateway from each node 
At the time t0, each node sends packets to the gateway of 1000 bytes in size, with inter-departures of $0.005 \mathrm{~ms}$, this ensures that each node sends the same number of packets during 300 s in simulation time. At the end of the simulation, we estimate the percentage of the packets number received by the gateway from each node "fig.4".

According to the analysis of results "fig.4", we can see that the medium access is not fair to the nodes. There are even some nodes far from the gateway that are not able to send packets. The Nodes that take a large percentage of packets sending, these nodes are the majority that exist in the transmission range of the gateway especially their neighbors in a single hop.

The experience gave these results in "fig.4" that reflect the congestion problem:

In the MAC layer, the author of the work [3] has discussed the role of mechanism of Queue management in the congestion problem. We can see this in the results of "fig.4". For example, the nodes, far from the gateway in two hops and more, did not deliver the packets, on the other side, the adjacent nodes have transmitted large quantities. The traffic coming from the STAs connected to adjacent nodes to the gateway frequently arrive and cover an important share of queue. Therefore, the packets that arrive from distant nodes will be rejected by the saturated queue. The work [3] has noted the role of the time difference EIFS imposed by the DCF protocol of 802.11 standard that leads to the congestion problem. This allows us to explain that the node N12 "Fig.4" has not transmitted a large amount of packets even if it is an adjacent node to the gateway. With DCF protocol, the node N12 can receive a mass of erroneous packets which forced him to wait a considerable period of EIFS to send packets.

If a node wants to communicate with the gateway, it will send a RTS to the gateway, this latter responds with a CTS. Subsequently, the node sends its packets, and at the end of the transmission, the gateway in turn transmits an ACK to the nodes located in its transmission range, among them: the node N12, so it must wait an EIFS period for data transmission.

The researchers of work [4] mentioned that among the reasons of congestion problem, there are the aggressive flows which have a high rate of transfer because of their proximity to the node. These flows are frequently received by an adjacent node to the gateway and often occupy the node queue preventing the other flows. The packets transmitted by the nodes far from the gateway will carry out several jumps and will suffer delayed each time they were crossing a node

\section{THE STUDY OF THE CONTROL FORWARDING BROADCAST}

\subsection{Definition}

It is a mechanism of forwarding control of broadcast traffic by flooding, where each node decides to remove or relay a flood packet by itself, and that based on the information collected from the neighborhood of one and two hops.

This mechanism operates in two phases:

- Phase of neighbor discovery: it is a simple process of periodic exchange of "hello" messages and is deployed to allow each node to know its neighbors and their neighbors

- Decision phase to remove or permit the retransmission of the flood packet

\subsection{Description of our CBF:}

Recent study [1] has focused on improving the capacity of wireless mesh networks based on the $802.11 \mathrm{~s}$ standard significantly reducing the number of redundant packets flood. The results showed that there was a large improvement in throughput and delay especially with the scaling.

\subsection{The Performed Study}

The objective of the work [2], is to define the behavior of the network capacity by varying the inter-departure and size of packets in each node during each simulation, and by using the CBF mechanism.

Through the obtained results, the work was able to deduce the optimal values of the inter-departure/size of packets that allow having the highest amount of the network capacity, and to compare the results in networks adopting the CBF mechanism and without $\mathrm{CBF}$.

This work has defined the network capacity by: the number of packets sent by all nodes to the internet through the gateway during a time interval.

The result of the study is as follow:

TABLE I. Simulation Results with CBF [2]

\begin{tabular}{|c|c|c|}
\hline grid & Packet size & $\begin{array}{c}\text { inter-departure } \\
\text { between packets }\end{array}$ \\
\hline $\mathbf{4 x 4}$ & 1000 bytes & {$[0.0037 \mathrm{~ms}, 0.01 \mathrm{~ms}]$} \\
\hline $\mathbf{5 x 5}$ & 1000 bytes & {$[0.0037 \mathrm{~ms}, 0.01 \mathrm{~ms}]$} \\
\hline $\mathbf{6 x 6}$ & 1000 bytes & {$[0.004 \mathrm{~ms}, 0.009 \mathrm{~ms}]$} \\
\hline $\mathbf{7 x 7}$ & 1000 bytes & {$[0.0037 \mathrm{~ms}, 0.007 \mathrm{~ms}]$} \\
\hline $\mathbf{8 x 8}$ & 1000 bytes & {$[0.0037 \mathrm{~ms}, 0.01 \mathrm{~ms}]$} \\
\hline $\mathbf{9 x 9}$ & 1000 bytes & {$[0.004 \mathrm{~ms}, 0.01 \mathrm{~ms}]$} \\
\hline
\end{tabular}

From "table I", we deduce that to reach the maximum of the network capacity the inter-departure should be between $0.0037 \mathrm{~ms}$ and $0.01 \mathrm{~ms}$ for packets of 1000 bytes by using the CBF.

This result can be exploited in the resolution of the congestion problem.

The result of a network capacity WMN with CBF, that allows having approximately the same quantity of capacity for different inter-departures of packet, permits the resolution of the congestion problem. The implementation of an algorithm called MAG (Medium Access Guarantee) is necessary which can affect different inter-departures to each node, the values of these inter-departures will stay in the interval $[0.0037 \mathrm{~ms}, 0.01 \mathrm{~ms}]$ and depend of the number of hops to the gateway. The objective is to minimize the number of packets that are able to access the medium in the same time.

\section{THE MEDIUM ACCESS GUARANTEE}

The algorithm MAG runs periodically in each node after assigning the path from a node to the gateway. The goal of this mechanism is that each node can have a different interdeparture of packets relative to others.

In order to make the algorithm runs, it must be based on two parameters: the number of hops from node $\mathrm{N}$ to the gateway and the number of nodes that use the node $\mathrm{N}$ to reach the 
gateway. We can recover these parameters from the information of the hybrid wireless mesh protocol HWMP on proactive mode (RANN mechanism), which is compatible with the architecture containing a gateway [9].

\subsection{The Algorithm}

The algorithm runs after the assignment of the paths by the HWMP protocol. It is based on two steps:

The first step is to determine the two parameters: NBH the number of hops allowing to a node $\mathrm{N}$ to reach the gateway and NBP the number of nodes that can use the node $\mathrm{N}$ so that its packets arrive to the gateway. The NBH parameter is gotten from the routing table provided by the HWMP protocol, and also the counting of the number of RREQ that will cross the node $\mathrm{N}$, and this number will represent the NBP parameter. The two parameters will be changed periodically depending on the period of RANN.

The calculation of the inter-departure of packets in each node will be the second step. The algorithm is based on the interval of the inter-departures $[0.0037 \mathrm{~ms}, 0.01 \mathrm{~ms}]$ found in the study [2], and on the two parameters NBH and NBP. The calculating will be effected as follows:

The algorithm will use in each calculation of an interdeparture the constant "I" which is the deference between the boundaries of the interval $[0.0037 \mathrm{~ms}, 0.01 \mathrm{~ms}]$ :

$$
\begin{gathered}
I=0.01-0.0037 \\
I=0.0063 \mathrm{~ms}
\end{gathered}
$$

After that, the mechanism divide the time "I" to a short times " $\tau$ ":

$$
\tau=\frac{\mathrm{I}}{\mathrm{NBH}+\mathrm{NBP}}
$$

Finally, the node will function with the inter-departure of packet I_D:

$$
\text { I_D }=0.0037+\tau * \mathrm{NBP}
$$

The "Fig. 5" summarizes our algorithm.

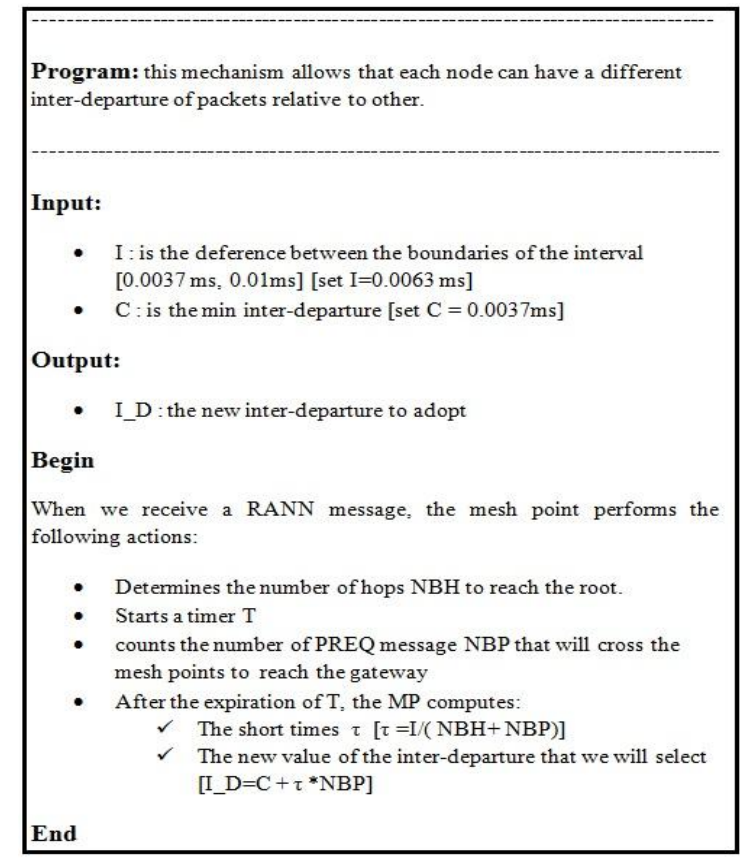

Fig 5: the algorithm of medium access guarantee

\subsection{The Results of Simulation with the Algorithm}

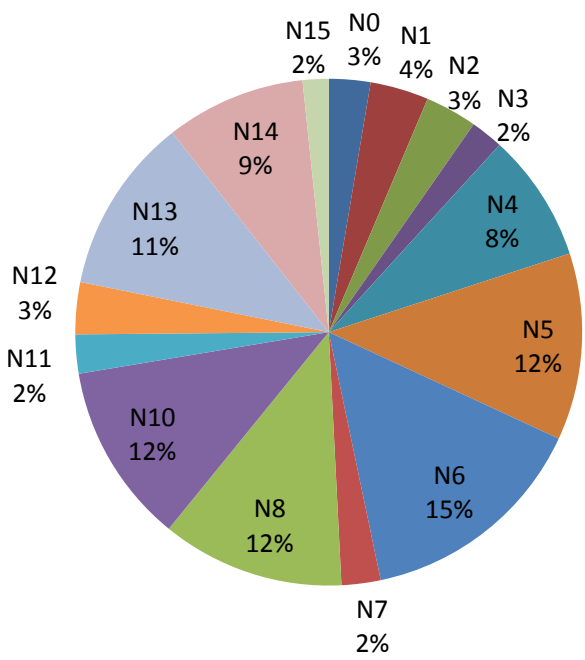

Fig 6: The percentage of the number of packets received by gateway from each node with algorithm

The simulation has been performed in the same environment, but this time the network nodes used the algorithm MAG. The "fig.6" presents the new percentage of each node packets which have reached the gateway.

\subsection{Analysis:}

Based on the obtained results, the gateway received more packets from far nodes N0, N1, N2, N3, N7, N11, N12, N14 and N15 that have suffered from a lack of resources. That allows us to deduce that the algorithm MAG has a positive effect on the node far from the gateway.

Consequently, the nodes near the gateway N5, N6, N8, N13 and N10 doesn't supply anymore the gateway with packets the same way as before.

The functioning of the algorithm MAG allows explaining the finding over it.

Regarding the first finding, the packets of far nodes experience a dispersion time when they cross the intermediate nodes, which increase the probability of accusing a delay to access the medium near the gateway. The MAG gives these nodes packets the shortest inter-departure, which allow them obtaining a higher flow rates, and then, having more chance to access the medium.

Concerning the second finding, the packets of the nodes near the gateway obtain higher inter-departures than the far nodes. So they obtain a flow rates lower and consequently undergo competitiveness to access the medium from packets of far nodes. Which explain largely the decrease of the number of these packets to reach the gateway.

The algorithm MAG has relieved pressure on the queues of the adjacent nodes to the gateway, which is why the packets of the far nodes have found more opportunity to the medium access and reach the gateway.

The quantity of packets sent by each node has been fixed during the time of simulation. The algorithm didn't consider this parameter, which have guaranteed the access of all the packets of different flows to the medium and to the gateway. 


\section{CONCLUSION}

This work allowed extracting some conclusions:

The DCF protocol used in wireless mesh networks is not efficient. In the first simulation, we have shown that this protocol does not guarantee to all packets of different nodes to access the medium, especially the packets of the distant nodes, which are exposed to a fierce competition from the packets of the nearest nodes to the gateway.

To guarantee the access of different flows to the medium, it is sufficient to control the flows rates from different nodes. The algorithm MAG demonstrated this concept.

The algorithm MAG is based only on the packets rates as parameter, which promoted the medium access of different flows but not in a fair way. To obtain this latter, the algorithm have to control the packets rates according to the distance between the nodes and the gateway and also the quantity of data sent at the time $\mathrm{T}$.

To upgrade this work in the future, we envisage putting in place a protocol which will assure the fairness. This protocol will inform each node of the needs of the bandwidth of all the nodes surrounding them and share it with a fair manner.

\section{REFERENCE}

[1] Y.Saadi, B.Nassereddine, S.Bennani, A.Maach. An adaptative approach to control broadcast traffic in wireless mesh networks based IEEE 802.11s, Nov 2012, Agadir, International Conference Complex Systems ICCS, pp 1-7. 978-1-4673-4764-8.

[2] S.Jounaidi, Y.Saadi, B.Nassereddine, A.Haqiq. GoodPut estimation in wireless mesh networks based IEEE 802.11s, 28-30 May 2014, Casablanca, the fifth International conference of the next Generation Networks and Services (NGNS), pp 187 - 193. 978-14799-6608-0

[3] Nagesh S. Nandiraju, Deepti S. Nandiraju, Dave Cavalcanti, Dharma P. Agrawal. A Novel Queue
Management Mechanism for IEEE 802.11s based Mesh Networks, 10-12 April 2006, Phoenix, International Performance Computing and Communications Conference IPCCC, 7 pp. - 168, 1-4244-0198-4.

[4] Nagesh S. Nandiraju, Deepti S. Nandiraju, Lakshmi Santhanam, Dharma P. Agrawal. A Cache Based Traffic Regulator for Improving Performance in IEEE 802.11s based Mesh Networks, 9-11 Jan 2007, Long Beach. CA, Radio and Wireless Symposium, pp 293 - 296, 1-42440445-2.

[5] Harish Kongara, Yogesh R Kondareddy, Prathima Agrawal. Fairness and Gateway Classification Algorithm (GCA) in Multihop Wireless Mesh Networks, 15-17 March 2009, Tullahoma, 41st Southeastern Symposium on System Theory (SSST), pages 77 - 81, 978-1-42443325-4.

[6] Malik Mehroze, Khalid Usmani, Faraz Ahsan, Sohail Asghar. Fairness Based Dynamic Routing Technique (FsBDRT) in Wireless Mesh Network, Research Journal of Information Technology. V5, December 2013, pages 97-103.

[7] Jorge L S Peixoto, Marcial P Fernandez, Luis F de Moraes. Improving Fairness in Wireless Mesh Networks, 29 February 2012, Saint Gilles. Reunion, The Eleventh International Conference on Networks, Pages: 175-180, 978-1-61208-183-0.

[8] Salim Nahle, Naceur Malouch. Fairness Enhancement in Wireless Mesh Networks, 10-13 December 2007, Columbia University New York, 3rd International Conference on emerging Networking EXperiments and Technologies (CoNEXT), Article No 30, 978-1-59593$770-4$

[9] The Working Group for WLAN Standards of the IEEE. HWMP Specification [online]. Available on: https://mentor.ieee.org/802.11/public/06/11-06-1778-01000s-hwmp-specification.doc . 2006-11. 